\title{
Structural evolution of lamellar surfactant-silica hybrids upon calcination
}

\author{
C. Henrist ${ }^{\mathrm{a}, *}$, I. García Cano ${ }^{\mathrm{b}}$, M.A. Rodríguez ${ }^{\mathrm{b}}$, A.H. de Aza ${ }^{\mathrm{b}}$, R. Cloots ${ }^{\mathrm{a}}$, \\ A. Rulmont ${ }^{\mathrm{a}}$, C. Vogels ${ }^{\mathrm{c}}$ \\ ${ }^{a}$ Laboratory of Inorganic Structural Chemistry, University of Liège, building B6, B-4000 Liège, Belgium \\ ${ }^{\mathrm{b}}$ Departamento de Ceramica, Instituto de Ceramica y Vidrio, C.S.I.C., Arganda del Rey, 28500 Madrid, Spain \\ ${ }^{\mathrm{c}}$ Solvay S.A., B-1120 Brussels, Belgium
}

\begin{abstract}
A lamellar mesostructured silica was subjected to a progressive heat treatment in order to study its structural evolution and the characteristics of the resulting calcined powder. By combining informations from several physical methods, i.e. TG-DTA, XRD, TEM and nitrogen adsorption, it has been possible to evidence the formation of very small particles of silica at a temperature around $450{ }^{\circ} \mathrm{C}$, exhibiting a very high value of aspect ratio, consequently to the template loss by combustion. By increasing the temperature above $530{ }^{\circ} \mathrm{C}$, the dehydroxylation promotes a decrease in the surface area, followed by the sintering process at higher temperature, which nearly annihilate the surface area of the particles.
\end{abstract}

(C) 2003 Elsevier Science Ltd. All rights reserved.

Keywords: A. Layered structures; A. Nanostructures; E. Heat treatment; XRD

\section{Introduction}

The idea of exploiting an ordered array of organic molecules to act as a template for the formation of a lamellar inorganic lattice is not new [1-8]. Usually these lamellar material are studied as green bodies since they loose the long-range ordering upon heating.

Thermal treatment of organic-intercalated lamellar silica can be envisaged in order to increase the surface area of the material for catalysis or surface tailoring purposes, or towards any substance to be adsorbed on the silica particles.

A study of the evolution of the properties of silica lamellar phases during heating appears to be very useful in order to precise the phenomena that take place, the temperatures when they occur, the resulting morphology, porosity, chemical composition, functional groups present at the surface and total surface area of the heat treated powders.

\footnotetext{
* Corresponding author. Tel.: +32-4-366-3438; fax: +32-4-366-

E-mail address: catherine.henrist@ulg.ac.be (C. Henrist).
} 3413 .

\section{Experimental procedures}

The lamellar silica is obtained as following. Dimethyldioctadecylammonium chloride (DMDODACl), is softly heated to melting then added under vigorous stirring to an $\mathrm{HCl}$ solution of concentration $1.8 \mathrm{~mol} / \mathrm{l}$ at $50{ }^{\circ} \mathrm{C}$. The medium turns turbid and iridescent as the lamellar mesophase of the DMDODACl-water system is formed. The mixture is allowed to equilibrate at $50{ }^{\circ} \mathrm{C}$ for one hour in order to reach a complete organization of the template molecules into an ordered lamellar mesophase. The precursor of the silica, TEOS (tetraethylorthosilicate), is then added under stirring to the previous mixture. The Si:DMDODACl ratio was adjusted to 8.9. The mixture is left to react at $50{ }^{\circ} \mathrm{C}$ for $24 \mathrm{~h}$. A solid phase is formed, which keeps the symmetry of the surfactant mesophase that serves as a template. It is filtered off, washed with de-ionised water and air-dried. The resulting structure is made of thin, flat layers of amorphous silica intercalated with the organic template used to drive the oriented polymerization of the silica precursor species (see Fig. 1). The thermal treatments were performed in a muffle oven in an open alumina crucible and heated 


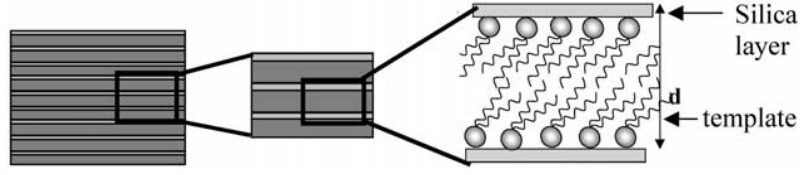

Fig. 1. Schematically representation of the structure of the lamellar silica intercalated with the template.

under air with heating ramp always set at $1{ }^{\circ} \mathrm{C}$ per minute.

A simultaneous thermal analysis was performed in a Netzsch apparatus, model STA 409, in order to obtain the TG-DTA data. The XRD patterns were obtained on a Siemens D5000 powder diffractometer equipped with a $\mathrm{Cu}$ radiation source and $\mathrm{Ni}$ filter. Transmission electron microscope imaging was performed on a Hitachi H700 transmission electron microscope working at 125 $\mathrm{kV}$ acceleration voltage. The specific surface measurements were carried out on a Monosorb ${ }^{\circledR}$ surface area analyzer from Quantachrome working with the single point method, which gives directly access to the value of the surface area.

\section{Results and discussion}

\subsection{Thermal analysis}

In an attempt to be as close as possible to the equilibrium conditions, a very slow heating ramp was used with capping the crucible with alumina powder and platinum lid [10]. The resulting thermogravimetry and differential thermal analysis are shown in Fig. 2.

The total mass loss at $1000{ }^{\circ} \mathrm{C}$ is $60 \%$ wt. The first weight percent loss is due to moisture desorption. As can be seen on the TG curve, the thermal degradation of the template begins starting from $150{ }^{\circ} \mathrm{C}$. It takes place in several successive steps that constitute a complex process. This effect is better evidenced by the derivative DTG curve where three well-separated peaks appear. The same profile can be observed in the DTA curve.
The quaternary ammonium nature of the template implies some Hoffman elimination or substitution reaction [11-13], followed by the pyrolysis and combustion of the degradation products. It can be seen in the DTA curve three peaks with maximum at $313{ }^{\circ} \mathrm{C}, 335^{\circ} \mathrm{C}$ and $480{ }^{\circ} \mathrm{C}$. All of these correspond to exothermic phenomena. These exothermal peaks appear to mask the endothermic dehydroxylation process that is expected to take place around $500{ }^{\circ} \mathrm{C}$. The total elimination of organic and $\mathrm{OH}$ groups from the silanols seems to be fully completed at $600{ }^{\circ} \mathrm{C}$.

\subsection{X-ray diffraction}

The typical XRD pattern of lamellar mesostructured silica is shown on Fig. 3 (curve a: "untreated material"). It exhibits two well-resolved peaks at low angle values that can be indexed as (001) and (002) reflections, therefore indicating a lamellar symmetry. These peaks correspond to a primary d-spacing of $38 \AA$ (see Fig. 1). In the wide-angle region, there is only a very broad peak relative to the $\mathrm{Si}-\mathrm{O}-\mathrm{Si}$ bonds period as well as the template alkyl chain crystallization. The absence of sharp peak in this region indicates that the silica sheets are amorphous in the bulk, and also assesses that the tem-

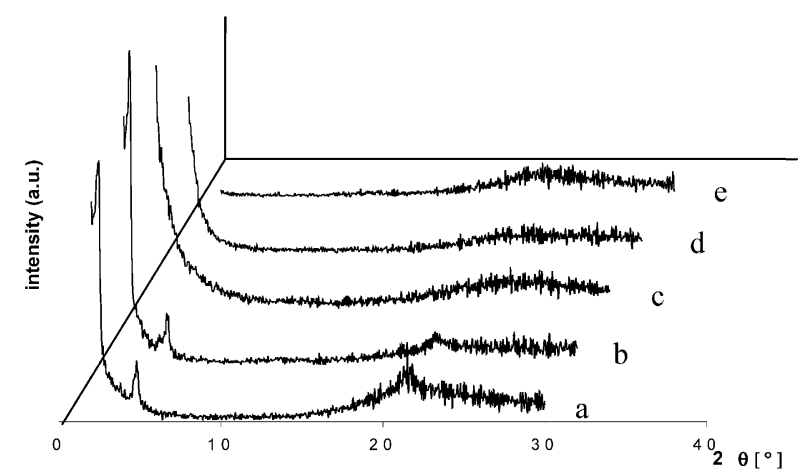

Fig. 3. XRD patterns of a lamellar mesostructured silicas: (a) untreated material, (b) heat treated at $150{ }^{\circ} \mathrm{C}$, (c) $240{ }^{\circ} \mathrm{C}$, (d) $825^{\circ} \mathrm{C}$, (e) $1000^{\circ} \mathrm{C}$.
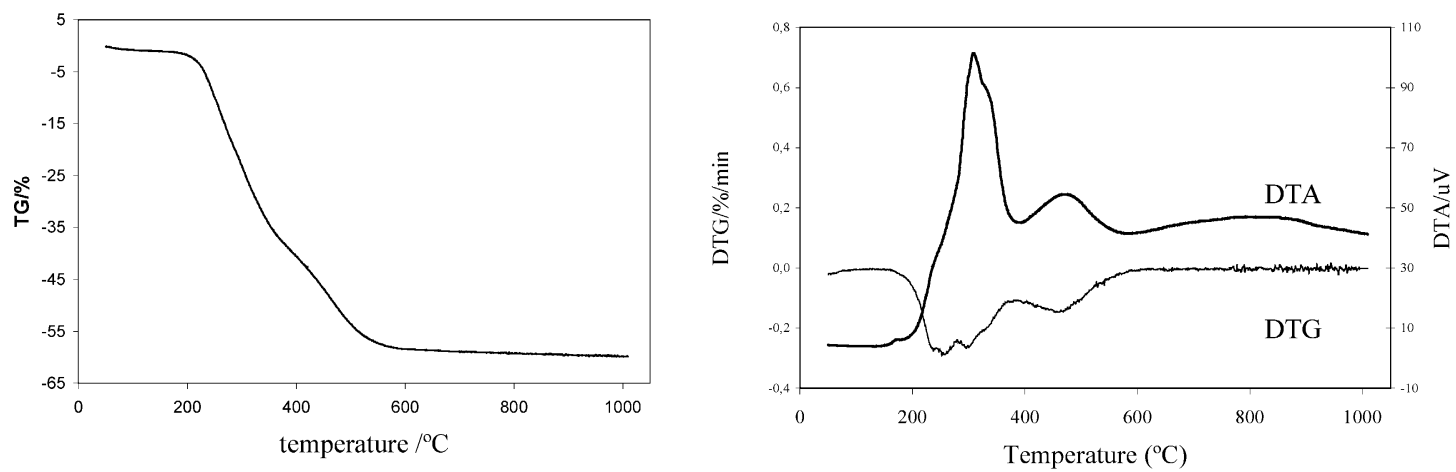

Fig. 2. Left: Thermogravimetry and right: differential thermal analysis (DTA) and thermogravimetry derivative (DTG) of the lamellar mesostructured silica. 
plate molecules have crystallized with very poor ordering. At a temperature of $150{ }^{\circ} \mathrm{C}$ (curve b), only a little water is expected to desorb but the overall structure is preserved. When increasing further the temperature, the thermal degradation of the organic moiety begins and gives rise to the total disappearance of diffraction pattern. After total combustion of the surfactant, the remaining silica can only undergo dehydroxylation, which does not promote any change in the diffraction pattern. This condensation step does not imply a crystallization of the dry silica. In conclusion, it can be said that the structure can remain almost intact up to $150{ }^{\circ} \mathrm{C}$, but is readily destroyed as the loss of the template occurs at higher temperature.

\subsection{Transmission electron microscope}

The morphology of the particles constituting the powders reveals the presence of large sheets of silica that appear to be extremely thin with poor rigidity, since folding or pleating of the lamellae can be observed (see Fig. 4A and B). After a thermal treatment at $650{ }^{\circ} \mathrm{C}$, such thin sheets of silica are more easily encountered than before calcination. It is an important result as it shows that the lamellae of silica do not undergo condensation one upon each other directly after the combustion of the surfactant. The thickness of the silica walls in untreated lamellar materials has been evaluated
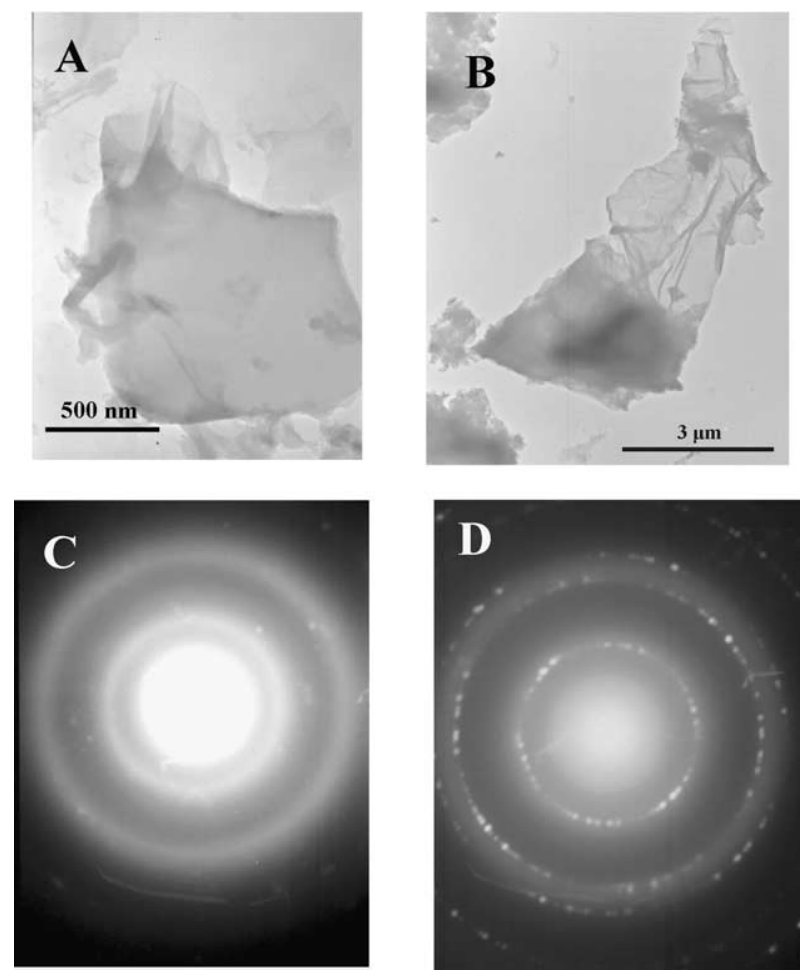

Fig. 4. TEM micrographs of lamellar mesostructured silica: (A) uncalcined material, (B) material calcinated at $650{ }^{\circ} \mathrm{C}$, and SAED patterns of (C) uncalcined material and (D) material calcinated at $650^{\circ} \mathrm{C}$. to $7-11 \AA[9,14,15]$. The diffraction rings that appear in Fig. $4 \mathrm{C}$ indicate that the untreated lamellar material is not well crystallized, as seen before in XRD pattern. The SAD electron diffraction pattern shown in Fig. 4D was taken on a nearly individual lamella of calcined silica and exhibits small diffraction spots, which indicates an enhancing of the crystallinity upon heating. This behavior could however not be general since no diffraction peak was observed by XRD on calcined material as a bulk powder.

\subsection{Nitrogen adsorption}

The specific area of samples was measured at each interval of temperature. The results are reported in Fig. 5. A very low specific surface area characterizes the untreated material, due to the fact that the interlayer region is completely filled with the template molecules. The structure remains intact up to $150{ }^{\circ} \mathrm{C}$, at which the template begins to degrade and burn. The gases evolved during this degradation go out of the interlayer space, therefore opening the layered structure and giving access to more nitrogen adsorbate. This is evidenced by a sudden jump of the specific area. As the combustion proceeds, the specific surface area reaches values as high as $700 \mathrm{~m}^{2} / \mathrm{g}$ for the sample calcined at $450{ }^{\circ} \mathrm{C}$, then begins to decrease at higher temperature. This is explained by the dehydroxylation $(\mathrm{Si}-\mathrm{OH}$ condensation) phenomenon of the remaining silica. At the temperature of $875^{\circ} \mathrm{C}$, the sintering must have begun as the surface area dramatically dropped to $1.8 \mathrm{~m}^{2} / \mathrm{g}$, which corresponds to big aggregates without valuable porosity. The total disappearance of hydroxyl groups at this temperature is in agreement with the TGA data and was also checked by infrared spectroscopy (not shown here). Regarding these results and using a simple model of square flat particles of pure silica, it is possible to simulate the surface area of powders constituted by platelets exhibiting various sizes $(a)$, thickness $(h)$ and aspect ratios

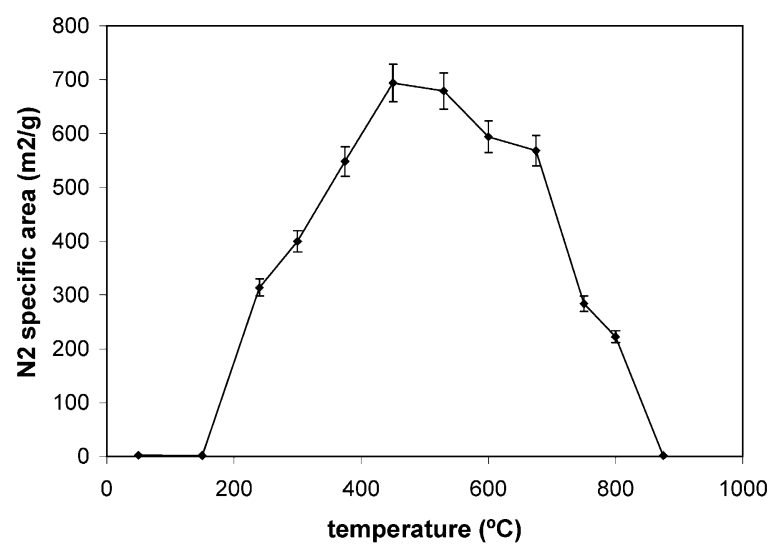

Fig. 5. Evolution of the specific area determined by BET nitrogen adsorption of a lamellar mesostructured silica with the temperature. 


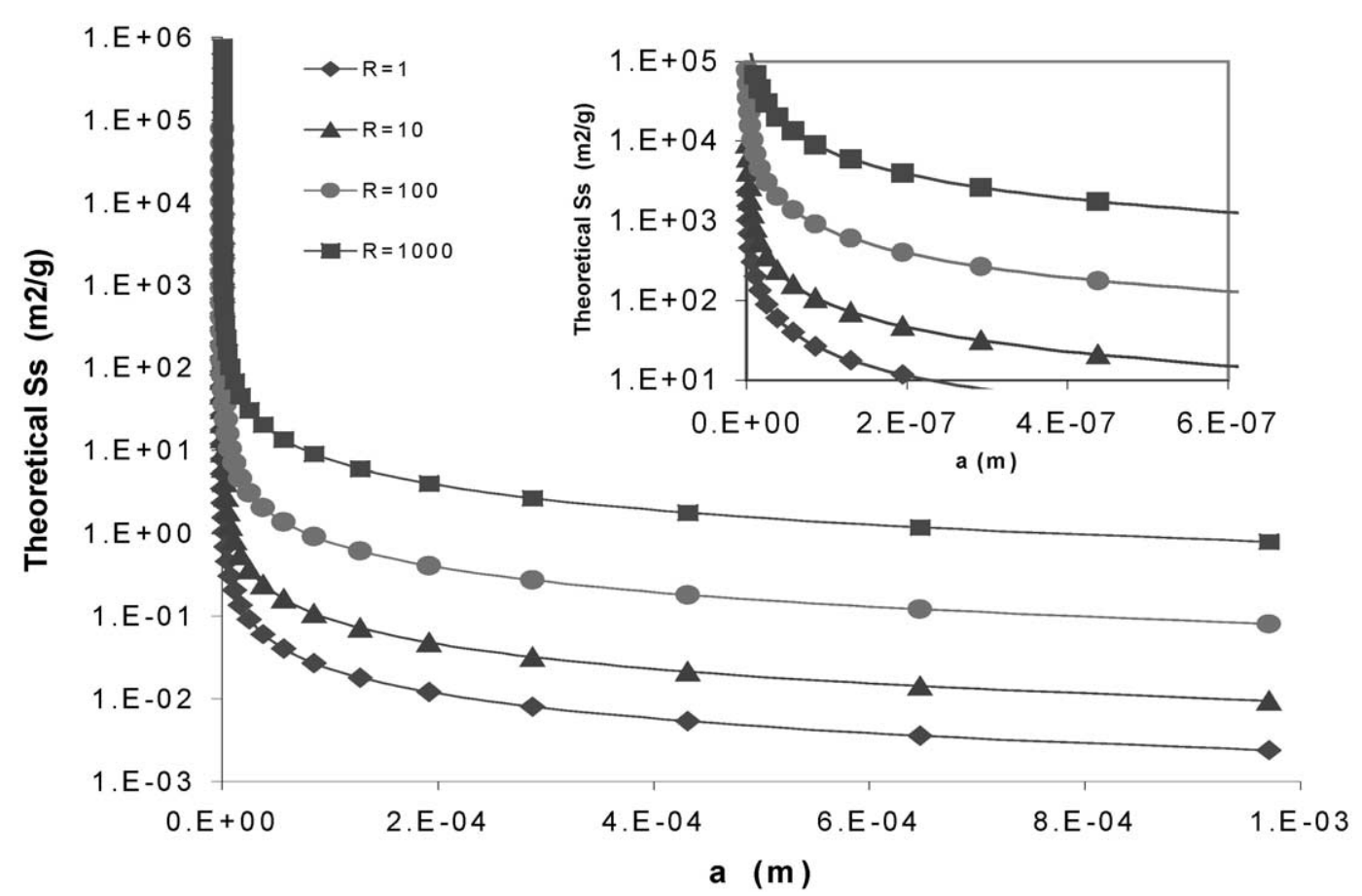

Fig. 6. Surface area of silica powder with respect to the size of the particles (a) and their aspect ratio $R$. Inset: zoom at the very small values of $a$ (from 0 to $60 \mu \mathrm{m}$ ).

$(R=a / h)$, assuming that the particles are monodisperse with a bulk density $(d)$ of $2.610^{6} \mathrm{~g} / \mathrm{m}^{3}$. It is easy to calculate that the specific surface area of a powder made of silica platelets can be expressed by Eq. (1):

$S_{\mathrm{s}}=\frac{S}{m}=\frac{2 a^{2}+4 a h}{a^{2} \cdot h \cdot d}=\frac{2 \cdot(a+2 h)}{a h d} \mathrm{~m}^{2} / \mathrm{g}$

Fig. 6 shows the simulation of the surface area of such silica powders with respect to the size of the particles $(a)$ and the aspect ratio $(R=a / h)$. The size required for a surface area of $700 \mathrm{~m}^{2} / \mathrm{g}$, corresponding to the sample calcined at $450{ }^{\circ} \mathrm{C}$, can be evaluated for each aspect ratio value. Referring to Table $1,1 \mu \mathrm{m}$-large silica lamellae should have an aspect ratio around 1000, which means a thickness of the order of $11 \AA$. This corresponds to $\cong 2$ silica molecular layers.

\section{Discussion}

Considering all experimental results jointly, behavior of lamellar mesostructured silica under thermal treatments can be described as follows: The untreated material is hardly non-porous. Decomposition and elimination of the organic moiety under heating take place in a complex way as shown by the bimodal effect in DTG and DTA (Fig. 2) between $200{ }^{\circ} \mathrm{C}$ and $400{ }^{\circ} \mathrm{C}$. Simultaneously, a big increase in specific surface is observed. This process leads to materials with very high specific surface, up to $700 \mathrm{~m}^{2} / \mathrm{g}$ at $450{ }^{\circ} \mathrm{C}$. Also in this
Table 1

Evaluation of the particle size required for each value of aspect ratio, giving a surface area of $700 \mathrm{~m}^{2} / \mathrm{g}$

\begin{tabular}{lc}
\hline Aspect ratio $R$ & Size of particles required for $\mathrm{Ss}=700 \mathrm{~m}^{2} / \mathrm{g}$ \\
\hline 1 & $3.3 \mathrm{~nm}$ \\
10 & $13.2 \mathrm{~nm}$ \\
100 & $112 \mathrm{~nm}$ \\
1000 & $1.1 \mu \mathrm{m}$ \\
10000 & $11 \mu \mathrm{m}$ \\
\hline
\end{tabular}

temperature range a decreasing in ordering is observed by XRD. Above $450{ }^{\circ} \mathrm{C}$, dehydroxylation is accompanied by a decrease of the specific surface. After this temperature the sintering between the silica sheets is predominant as shown by the strong decreasing in the specific surface. During all treatment no morphological changes are observed by TEM on the silica sheets. Only an increase of the crystallinity of the sheets can be deduced from SAD when temperature rises.

\section{Conclusions}

The structural and chemical evolution of lamellar mesostructured silica was studied under thermal treatment. The untreated material is hardly non-porous and exhibits a long-range order. At a temperature of $450{ }^{\circ} \mathrm{C}$, 
the powder is believed to be constituted by numerous, very thin silica sheets with a high value of aspect ratio. Above this temperature, the surface area of the sample decreases because of sintering process. Regarding all these results, it can be said that calcination of a lamellar mesostructured silica at a temperature around $450{ }^{\circ} \mathrm{C}$ is a simple way to produce a silica substrate with huge specific surface area and high value of aspect ratio of the particles.

\section{Acknowledgements}

This work was partly supported by Solvay S.A., Belgium, by the F.N.R.S. (Belgian National Fund for Scientific Research) and by the Spanish Science and Technology agency (CICYT) trough the project MAT 2000-0941.

\section{References}

[1] Laversanne R. Macromolecules 1999;25:489-91.

[2] Dubois M, Gulik-Krzywicki TH, Cabane B. Langmuir 1993; 9:673-80.

[3] Ogawa M. Langmuir 1997;13:1853-5.

[4] Yun SK, Maier J. InorgChem 1999;38:545-9.

[5] de Farias RF, Airoldi C. J Solid State Chem 2000;149:113-9.

[6] Sayari A, Moudrakovski I, Reddy JS. Chem Mater 1996;8:2080-8.

[7] de Farias RF, Airoldi C. Colloids and Surfaces A: Physicochemical and Engineering Aspects 2000;172:145-52.

[8] Ogawa M. Supramolecular Science 1998;5:247-51.

[9] Kresge CT, Lonowicz M, Roth WJ, Vartuli JC, Beck JS. Nature 1992;359:710.

[10] Paulik F, Paulik J. Thermochimica Acta 1986;100:23-59.

[11] Abdallah DJ, Weiss RG. Chem Mater 2000;12:406-13.

[12] Verrall RE, Burns JA. Can J Chem 1974;52:3438.

[13] Xie W, Gao Z, Liu K, Pan W-P, Vaia R, Hunter D, Singh A. Thermochimica Acta 2001;367-368:339-50.

[14] Van den Bossche G, Sobry R, Fontaine F, Clacens J-M, Gabelica Z. J Appl Cryst 1997;30:1065-74.

[15] Henrist C, Rulmont A, Cloots R. Key Engineering Materials 2002;206-210:1981-4. 